\title{
Reliable broadband wireless communication for high speed trains using baseband cloud
}

\author{
Qinglin Luo ${ }^{1 *}$, Wei Fang ${ }^{1}$, Jinsong $\mathrm{Wu}^{1}$ and Qingchun $\mathrm{Chen}^{2}$
}

\begin{abstract}
We propose a novel high-speed train communication using baseband cloud (C-HSTC) system framework for providing continuous broadband services to highly mobile users. This framework is featured with a new virtualized single cell design which mitigates the impact of conventional handover failures and guarantees continuous communication services. Through exploiting the baseband units (BBU) cloud and the ful frequent frequency reuse in the virtualized single cell, we also proposed a highly efficient joint transmit beamforming algorithm targeting at compensating the inter-carrier interference (ICI) caused by severe Doppler frequency shift due to mobility. Numerical analysis shows that the new architecture and corresponding algorithms are suitable for high-speed train communication and can provide a continuous data rate of more than 100 megabits per second (Mbps) for passengers at a speed of 450 kilometers per hour ( $\mathrm{kmph}$ ). This would help to achieve satisfactory mobile broadband services for high speed train passengers.
\end{abstract}

\section{Introduction}

Mobile broadband communications have been extensively developed for terrestrial transportation to respond to the increasing traffic of online multimedia, gaming, mobile application downloading. However, without changes, those mobile communication technologies may not be suitable for the needs of data-intensive communications for high speed train passengers, since the relevant moving speeds are much higher and more challenging for communication designs, for example, the China Railways High-speed (CRH) train between Beijing and Shanghai at a speed of 350 kilometers per hour $(\mathrm{kmph})$. In recent years, numerous efforts have been made on adapting the conventional Global System for Mobile Communications (GSM) framework to High-Speed Train Communication (HSTC) which gives birth to the railway specific GSM standard, GSM-R. In the high speed train scenario, although GSM-R has been so successful in voice communications [1], it cannot support high-rate broadband data services, such as online video or gaming, for a train at a speed up to $500 \mathrm{kmph}$.

This paper presents a framework on high data rate communications for passengers on high-speed train. Our

\footnotetext{
* Correspondence: Qinglin.a.Luo@alcatel-sbell.com.cn

'Bell Laboratories China, Room D400, Building 3, No. 388, Ningqiao Road,

Pudong New District, Shanghai 201206, People's Republic of China

Full list of author information is available at the end of the article
}

proposed scheme is capable of providing continuous broadband service for users on high mobility by reducing the signal degradation caused by frequent handover failures and high Doppler frequency spread.

\section{Previous work}

Two major challenges have been identified for providing mobile high-rate data services to passengers on the highspeed train: 1) too frequent handover which causes a high call drop rate and quick battery drain; and 2) poor link quality due to high Doppler spread.

To reduce the call drop rate, conventional GSM-R based HSTC systems require base stations to be densely deployed along railway tracks, and adjacent cells to have a large overlapped area so as to guarantee sufficient handover time [2]. Full functional base stations can be replaced by distributed antenna systems (DAS), radio-over-fiber (RoF) systems [3,4], or remote radio heads (RRH) [5] for cost saving and network topology simplification.

A radio-over-fiber (RoF) [3] based solution for broadband railway communication consists of several radio access units (RAU) located along the rail tracks, and an optical ring network interconnects them. The radiofrequency (RF) band signals transmitted by a base station are firstly carried by an optical fiber to the RAUs, and then transmitted into the air. Commonly, each RAU has its fixed radio frequency. A range of issues related to 
RoF in HSTC systems including the handover [4], coverage efficiency [6], multi-mode multi-band accessing [7], and field measurement and analysis [8] have been addressed in the literatures. The major challenge of the RoF based HSTC system is that it requires frequent band switching and complex optical routing which restricts the length of the coverage of a base station.

Remote radio head (RRH) based solution as shown in Figure 1 is a more favorable evolution. RRHs have been widely deployed in cellular systems, and the RRH based HSTC solution relies on a fiber network to convey signals along the tracks, similar to the RoF system, but the signals running on the fiber, i.e. a Common Public Radio Interface (CPRI), is not RF-band signals but baseband I/Q data symbols so as to avoid RF-band signal degradation due to long-distance propagation. Theoretically, the length of a CPRI link is unlimited, while in practice, it is only constrained by the timing errors accumulated along the propagation [9]. RRH-based HSTC systems have been presented in [5].

The analysis in [2] based on a conventional GSM-R system shows that the handover process in such a system requires multiple seconds to complete, thus the coverage efficiency (effective coverage area per radio head) can become prohibitively low with the increase of train speed.

With the introduction of Long Term Evolution (LTE) systems to high-speed train communications [10], the handover delay could be significantly reduced to less than 1 second [11]. Due to the limited uplink resource and inadequate uplink scheduling, the LTE system may still suffer from frequent handover failures caused by unreliable uplink measurement reports at high mobility. Furthermore, for a train with hundreds of passengers, a large amount of handover requests in a short time may easily leads to signaling congestions. To address this issue, there are numerous interests in adopting LTE-based mobile relays dedicated for high speed train scenario [12] where user traffics are aggregated by a mobile relay fixed on the top of a train carriage and then communicated to the track side base stations (BS). The system only needs to maintain one high-mobility link between the mobile relay and the track-side BS, e.g. the mobile backhaul, instead of hundreds of individual access links. Mobile relay can reduce the possibility of signaling congestions, but it still requires a large overlapped cell boundary area for smooth handovers.

Doppler spread is another major challenge for realizing wireless broadband high speed train communications. Especially for HSTC system based on orthogonal frequency division multiplexing (OFDM), the fast channel variations within one OFDM block destroy the orthogonality between subcarriers resulting in inter-carrier interference (ICI) proportional to the Doppler frequency. In $[13,14]$, the authors established a compact model of ICI channel with a scalable parameterization, and proposed new receivers capable of ICI cancellation based on the ICI channel estimation. The authors of [15] proposed a time-domain windowing operation in the transmitter and receiver to form nulls around a subcarrier in frequency domain so as to reduce the ICI.

Recently, Doppler compensation via exploiting multiple antennas has attracted a lot of attention. [16] proposed to combine the received signals from each antenna with regard to the ICI power levels, while [17] tried to form virtual stationary antennas by using spatial interpolation to mitigate Doppler spread.

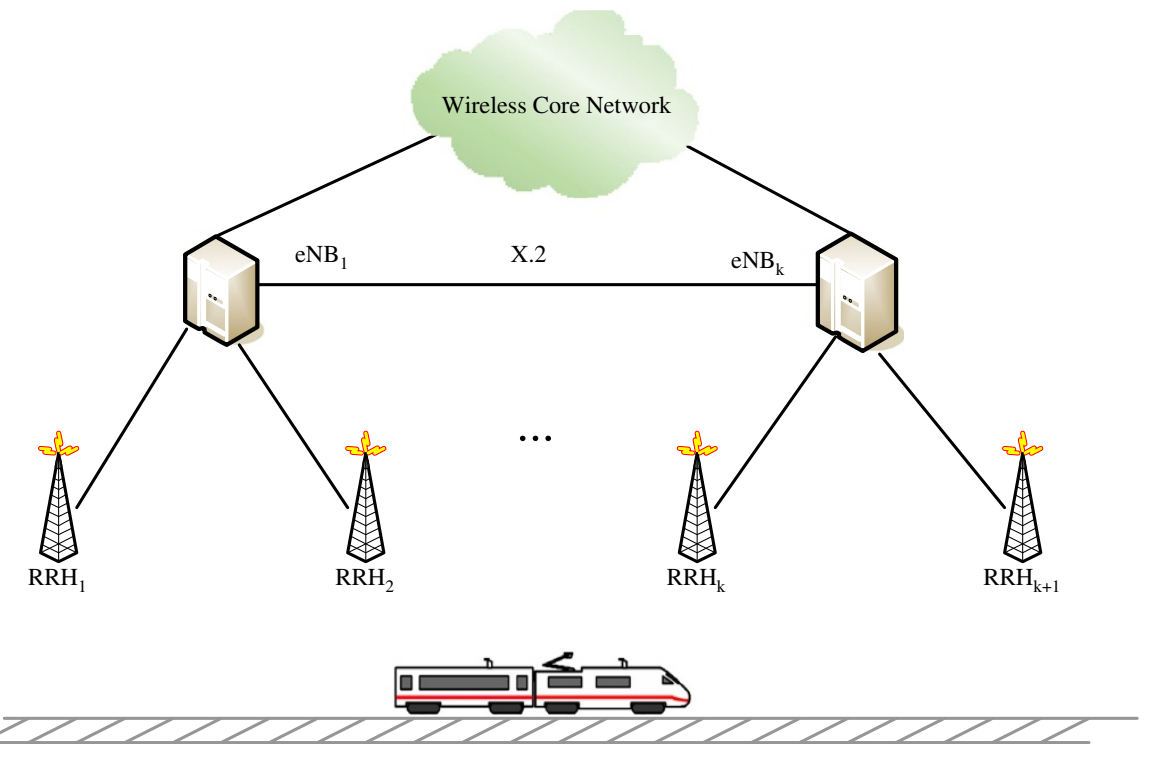

Figure 1 Conventional HSTC system based on RRHs. 
The performance of above approaches is generally good, but it has been noted that all the approaches are enhancements to receiver side and inevitably increase receiver complexity.

\section{Primary contributions}

In this paper, we consider a new HSTC system framework featured with both a cloud based architecture and a joint transmit beamforming scheme capable of Doppler compensation. We firstly present a novel virtualized single cell design characterized by a large number of RRHs associated to a baseband unit (BBU) cloud. The RRHs are allocated with the same set of radio resources including the cell ID, the frequency band, and reference signals, thus they are in full frequency reuse. The serving RRHs are dynamically selected based on the train's instantaneous geographical information, and perform cooperative transmission so as to avoid the inter-RRH co-channel interference and achieve a higher data rate.

With such a design, the necessity of the conventional handover is removed at the cost of inter-RRH interference. We propose to employ joint transmit beamforming to combat the inter-RRH interference. We found that the inter-carrier interference (ICI) due to Doppler spread can be compensated at the transmitter side with a new transmit beamformer design. We formulate an optimal joint transmit ICI beamforming problem, and give the solution which maximizes the signal-to-ICI power ratio of a high mobility link subject to a total transmission power constraint over multiple antennas.

The primary contributions of the proposed scheme are summarized as follows:

(1) A new HSTC architecture is proposed to realize handover-free high speed train communication. The trackside network is based on a BBU cloud which enables a new virtualized single cell design and geoaided fast RRH selection scheme. This architecture leads to a HSTC network with full frequency reuse and requires joint processing among distributed RRHs.

(2) A new joint transmit beamforming scheme is used to avoid the co-channel interference (CCI) caused by full frequency reuse and mitigating the intercarrier-interference (ICI) caused by Doppler spread.

The effectiveness of the two features is interdependent. The BBU cloud and virtualized single cell architecture provides infrastructure basis for the joint transmit beamforming, while the joint beamforming algorithm effectively exploits the full frequency reuse and mitigates the impact of Doppler frequency shift. Our numerical evaluation shows that continuous data rate of more than 100 megabits per second (Mbps) can be achieved for passengers at a speed of $450 \mathrm{kmph}$. This would guarantee continuous data-intensive services for today's high speed train passengers.

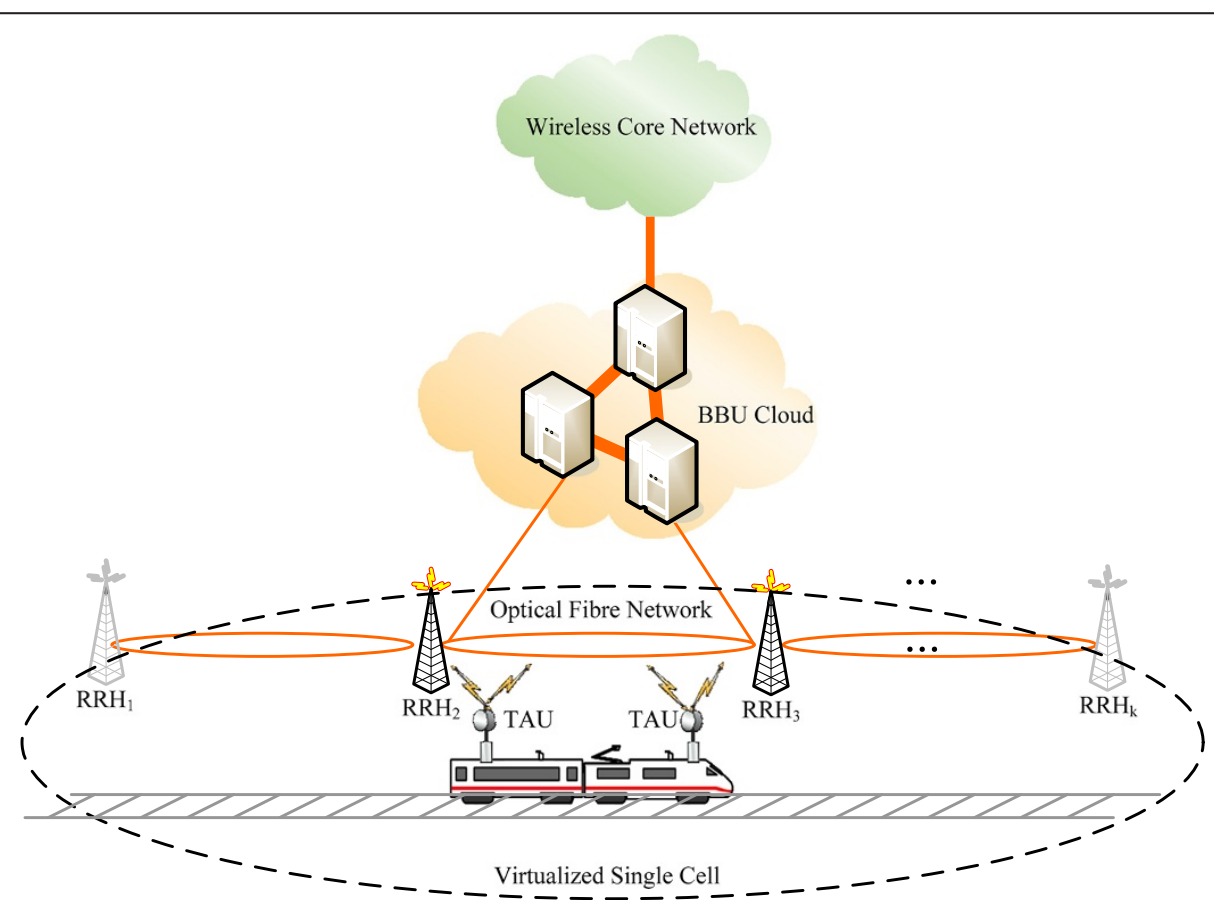

Figure 2 Cloud-based high-speed train communication system architecture. 


\section{C-HSTC system architecture}

Figure 2 shows the new architecture of an HSTC system based on a baseband cloud (C-HSTC). The system consists of onboard train access units (TAU), trackside RRHs, and a BBU cloud.

The TAU is analogous to a mobile relay, responsible for gathering user traffic from/to the access points deployed in the carriages and communicating with the trackside RRHs via the air-interface channel. The number of TAUs equipped on a train depends on the user traffic volume and the train-to-ground transmission bandwidth. Typically, two TAUs may be deployed: one on the front and the other at the end. This reduces the opportunity of interference between TAUs, while providing sufficient spatial diversity for the trackside RRHs to exploit advanced multiple-in-multiple-out (MIMO) transmission technologies. An exemplary system will be evaluated in the sequel of this paper. The TAUs and the in-train access points are connected by optical fiber so as to provide bandwidth for broadband services and keep the latency low.

The trackside RRHs have limited capability of processing radio and intermediate frequency signals. Each RRH can have multiple transmit and receive channels, for instance, up to eight channels for a Time-Division Long Term Evolution (TD-LTE) RRH. They are connected to BBUs via optical fiber interfaces such as common public radio interface (CPRI). Various topologies, such as star, chain, tree, and ring can be supported by the CPRI. In the context of HSTC where linear coverage is desired, RRHs can be connected by an optical network with a hybrid topology for further extension, as shown in Figure 2.

Unlike a conventional HSTC system where BSs are deployed distributedly along the track and connected via a low speed interface such as $X 2$ [1], the new architecture consists of a pool of collocated BBUs connected by fast data links and under the control of a common resource scheduler, e.g. a BBU cloud as shown in Figure 3.

In the cloud, BBUs can support different communication protocols such as GSM, UMTS, LTE Time Division Duplex (TDD), LTE Frequency Division Duplex (FDD), etc. Their computing resources are under the supervision of a global scheduler/controller, thus can be dynamically allocated to different TAUs according to their instantaneous requirements. Furthermore, there is a unified input/ output (I/O) interface between the BBU cloud and the $\mathrm{RRH}$ network, which is responsible for addressing and switching signals between the RRHs and BBUs. The addressing and switching is configurable not only statically via the operation and management (OAM) interface, but also dynamically by a load balancer which is monitoring the BBU computation resources. Unlike a conventional HSTC system where each BS has its own timing source, the entire cloud has a common timing source which helps reduce the interferences in the C-HSTC network due to timing misalignments.

\section{Geo-aided fast RRH selection}

Instead of relying on handovers to switch connections between the base stations as in the conventional system, we consider to implement a fast RRH selection scheme enabled by the cloud-HSTC architecture.

Unlike a normal cellular system where user location and speed are both arbitrary, an HSTC system serves for a train whose track is fixed and speed is easily known. Since all RRHs are centrally controlled by a BBU cloud in the new architecture, the train's geographical information (location and speed) is traceable in nearly real time. Only the RRHs that the train is approaching need to be selected and powered up for transmission. Other RRHs can be powered down for energy saving and interference reduction, as shown in Figure 4.

Two alternative approaches can be employed to obtain the geographical information of a train. The first one is based on a dedicated control channel within the trainto-ground network, which utilizes more powerful error control mechanisms such as $1 / 3$ rate turbo code, and a lower order modulation scheme such as QPSK modulation. It trades the data rate for a relatively more reliable data tunnel for the transmission of geographical information via the mobile backhaul to the BBU cloud. The geographical information is obtained from the GPS receiver equipped on the train. The BBU cloud determines which RRHs would be selected for transmission upon the receipt of the geographical information.

Alternatively, sensors can be deployed along the tracks to detect and report the geographical information of a train. When a train passes by a sensor, it triggers the sensor to measure the speed and location of the train and report the information to a controller in the BBU cloud. The BBU selects and switches the RRHs for transmission based on the report. This method requires to maintain an extra sensor network, but it eliminates the need of a dedicated control channel and thus the resources can be utilized more efficiently for user traffic transmission. It is more suitable for scenarios where a sensor network is employed in the high-speed railway system for track maintenance and automatic train control. A recent work has included high-speed railway systems integrated with Communication-Based Train Control (CBTC) technology [18] for the sake of obtaining accurate information of speed and position.

\section{Virtualized single cell}

In addition to the fast RRH selection, we propose to implement a virtualized single cell based on the C-HSTC architecture to avoid the signaling overhead and delay 


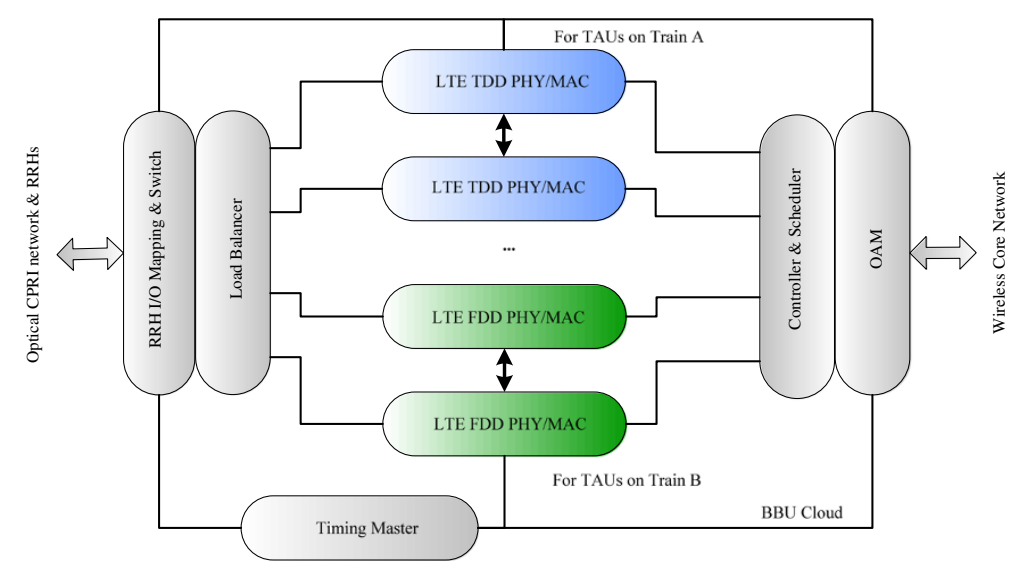

Figure 3 Example of a BBU cloud.

caused by frequent resource allocation and reallocation in a conventional system. The relevant designs include:

\section{(1) Centralized Scheduling in the BBU Cloud}

All BBUs in the cloud are connected with fast data links, which would be less than 1 microsecond. Therefore, the delay incurred by inter-BBU interaction becomes negligible. Furthermore, since the number of TAUs equipped on a train is limited, the resources for each TAU can be statically/semi-statically scheduled. Hence, instantaneous centralized resource scheduling at the cloud level is feasible and the scheduling delay is negligible.

\section{(2) Single Frequency Network with the Same Cell ID}

All RRHs share the same cell identification. Other configuration parameters bounded to the cell ID are also shared among the RRHs, including carrier frequency, reference signal resources, cell-specific scrambling codes, and so on. Then, the cell specific resources serving an onboard TAU do not need to be switched during the moving within the virtual cell.

\section{(3) Reference Signal Allocation}

Cell specific reference signals (CRS) are used for channel measurement and data demodulation by communication systems such as LTE. In the case that we adopt an LTE Release 8 or 9 system with limited number of CRS ports, CRS port allocation for RRHs along the tracks would inevitably be critical for interference control in a conventional system. In the virtualized single cell design, all RRHs associated to a cloud can be configured with the same set of CRS ports. The CRS signals transmitted by different nodes will be combined naturally over-theair. A joint transmission scheme is employed to ensure the backward compatibility, as well as coverage enhancement. RRH selection scheme is employed for reducing CRS interferences from irrelevant RRHs.

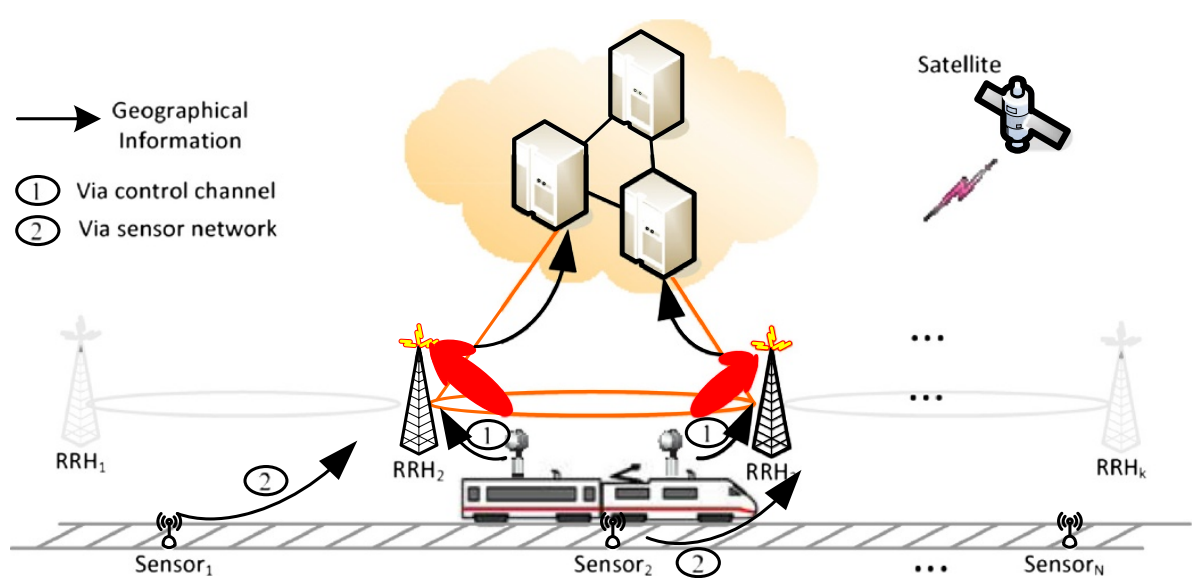

Figure 4 Geo-aided Fast RRH Selection. (a) $2 \times 2 \mathrm{MIMO}$ (b) $4 \times 2 \mathrm{MIMO}$ (c) $8 \times 2 \mathrm{MIMO}$ (d) $12 \times 2 \mathrm{MIMO}$. 
In LTE Release 10, the functionality of CRS is replaced by channel state information reference signal (CSI-RS). Only one CSI-RS configuration is allowed for each cell. At most 8 individual transmit antenna ports can be supported. However, the number of antenna ports required by all selected RRHs can exceed 8 when each RRH can be equipped with multiple antennas in the HSTC network. Thus, the antenna ports of CSI-RS configurations should be firstly mapped to the physical RRHs, and then to the physical antennas. Similarly, a joint transmission scheme is needed in case that multiple RRHs are selected for simultaneous transmission.

\section{(4) Channel State Information (CSI) Feedback}

The CSI-RS configuration corresponding to the selected RRHs is indicated by the BS with high layer signaling to the user equipment (TAU), and it might be valid for different sets of RRHs while the train is moving. For achieving better robustness, the measure/report $\mathrm{RRH}$ set should be larger than the transmission RRH set. Thus, unlike in a conventional LTE system where UE feedback is used by default for all the configured ports, UE feedback for part of the configured CSI-RS ports should be supported.

\section{Joint transmit beamforming based on virtualized cell}

We discussed the C-HSTC system architecture in the previous section. With such architecture, handovers would be only needed at the boundary of two virtual cells which is much infrequent compared with that in a conventional cell based system. Thus, call drops due to frequent handover failures can be significantly reduced. However, it should be noted that, since RRHs attached to the cloud now share the frequency band, inter- $\mathrm{RRH}$ interference would be necessary to be considered in the design. Furthermore, the link quality drop issue due to severe Doppler frequency shift still exists.

In the following, we first establish a signal model for OFDM systems under high mobility, then describe a simple joint transmit beamforming scheme capable of efficient Doppler compensation under the C-HSTC architecture.

\section{OFDM signal model under high mobility}

We first consider a conventional OFDM system with single transmit and receive antenna where $N$ complex symbols, $\mathbf{u}=\left[u_{1}, u_{2}, \ldots, u_{N}\right]$, are modulated onto $N$ orthogonal subcarriers by using an $N$-point IFFT. Assume that a cyclic prefix longer than the length of the channel impulse response is added to the signal to avoid inter-symbol-interference. The signals are assumed to be transmitted through a wide sense stationary time varying multipath channel consisting of uncorrelated paths with attenuation $h_{l}(t)$ and delay of $\left\{\tau_{l}\right\}$ with an impulse response

$$
h(t, \tau)=\sum_{l=0}^{L-1} h_{l}(t) \delta\left(\tau-\tau_{l}\right)
$$

Then the baseband received signal in time domain can be expressed as

$$
r(t)=\sum_{n=0}^{N-1} H_{n}(t) e^{j 2 \pi n f_{s} t} u_{n}+n(t)
$$

where $H_{n}(t)=\sum_{l} h_{l}(t) e^{-j 2 \pi n f_{s} \tau_{l}}$ is the channel frequency response of subcarrier $n$ at time $t, f_{s}$ is the subcarrier spacing and $n(t)$ is the AWGN with variance $\sigma_{n}^{2}$ at the receive antenna. Specifically, $H_{n}(t)$ can be approximated by using Taylor series expansion around $t_{0}$ up to the first-order term as [19]

$$
\begin{aligned}
H_{n}(t)= & H_{n}\left(t_{0}\right)+H^{\prime}{ }_{n}\left(t_{0}\right)\left(t-t_{0}\right)+O\left(\left(t-t_{0}\right)^{2}\right) \\
& \approx H_{n}\left(t_{0}\right)+H^{\prime}{ }_{n}\left(t_{0}\right)\left(t-t_{0}\right)
\end{aligned}
$$

Consequently, $r(t)$ can be approximated as

$$
r(t) \approx D(t)+I(t)+v(t)
$$

where $D(t)=\sum_{n=0}^{N-1} H_{n}\left(t_{0}\right) e^{j 2 \pi n f_{s} t} u_{n}$ is the desired part of the signal, and $I(t)=\sum_{n=0}^{N-1}\left(t-t_{0}\right) H_{n}^{\prime}\left(t_{0}\right) e^{j 2 \pi n f_{s} t} u_{n}$ is the ICI generating part of the signal.

\section{Joint transmit beamforming with Doppler compensation}

We consider the joint transmission of OFDM signals with a number of selected RRHs equipped with $K$ antennas in total. Signals from transmit antennas of different RRHs experience a different time varying channel to a receive antenna. However, there exists correlation among these channels due to the shared frequency band among RRHs, the same receive antenna, and the same velocity. This motivates us to exploit joint beamforming with different RRHs for coherent gain in the context of C-HSTC.

By employing channel estimation techniques provided in $[13,14]$, the trackside BBUs can estimate the channel correlation among the fixed part of the channels, $E\left[H_{j, n}^{H}\left(t_{0}\right) H_{k, n}\left(t_{0}\right)\right]$, the time-varying parts of the channels, $E\left[H_{j, n}^{\prime H}\left(t_{0}\right) H^{\prime}{ }_{k, n}\left(t_{0}\right)\right]$, and also the cross-correlation among them, $E\left[H_{j, n}^{\prime H}\left(t_{0}\right) H_{k, n}\left(t_{0}\right)\right]$ where the expectation is taken over the time and subcarriers, and they form the $K \times K$ matrices, $\mathbf{R}_{H H}, \mathbf{R}_{H^{\prime} H^{\prime}}$, and $\mathbf{R}_{H^{\prime} H}$, respectively. For a TDD system where uplink and downlink share the same band, the BBU can estimate the above information from the uplink channel. For an FDD system, this 
information can be obtained through downlink channel estimation and feedback.

In this context, unlike the conventional beamformer which targets at maximizing the signal to noise ratio (SNR) of the received signal, i.e. the maximum ratio transmission (MRT) scheme [20], we choose to design a timevarying beamformer of $\mathbf{w}(t)=\left[w_{1}(t), w_{2}(t), \ldots w_{K}(t)\right]^{T}$ for $K$ antennas targeting at maximize the ratio of signal to interference caused by time varying plus noise. The beamformed signal can be expressed as

$$
\mathbf{s}(t)=\mathbf{w}(t) u(t)
$$

The choice $\mathbf{w}(t)$ depends on the correlation among the channels and the computational complexity requirements induced. Note that the beamforming weights $\mathbf{w}(t)$ can be approximated by using Taylor series expansion as

$$
w_{i}(t)=w_{i}\left(t_{0}\right)+w_{i}^{\prime}\left(t_{0}\right)\left(t-t_{0}\right)+O\left(\left(t-t_{0}\right)^{2}\right)
$$

which forms the general beamforming weight vector of

$$
\mathbf{w}(t) \approx \mathbf{w}_{0}+\left(t-t_{0}\right) \mathbf{w}_{1}
$$

Using (7), appropriate beamforming weights can be investigated up to a desired precision and complexity. In this paper, we restrict ourselves to the approximation up to the first-order term. Thus, the received signal can be approximated as

$$
r_{p}(t) \approx D_{p}(t)+I_{p}(t)+n(t)
$$

where

$$
\begin{aligned}
& D_{p}(t)=\sum_{n=0}^{N-1} \mathbf{H}_{n}\left(t_{0}\right) \mathbf{w}_{0} e^{j 2 \pi n f_{s} t} u_{n} \\
& I_{p}(t)=\sum_{n=0}^{N-1}\left(t-t_{0}\right)\left[\mathbf{H}_{n}^{\prime}\left(t_{0}\right) \mathbf{w}_{0}+\mathbf{H}_{n}\left(t_{0}\right) \mathbf{w}_{1}\right] e^{j 2 \pi n f_{s} t} u_{n}
\end{aligned}
$$

For a given $\mathbf{w}_{0}$ and $\mathbf{w}_{1}$, the power of the desired signal part and ICI generating part of the signal and can be expressed as

$$
\begin{gathered}
P_{D}=E\left\{\operatorname{tr}\left(D_{p}(t)\right)\right\} \\
=E\left\{\operatorname{tr}\left(\mathbf{H}_{n}\left(t_{0}\right) \mathbf{w}_{0} \mathbf{w}_{0}^{H} \mathbf{H}_{n}^{H}\left(t_{0}\right)\right)\right\} \\
P_{I}=E\left\{\operatorname{tr}\left(I_{p}(t)\right)\right\} \\
P_{N}=\sigma_{n}^{2}
\end{gathered}
$$

And the desired signal to ICI ratio plus noise ratio (SINR) can be expressed as

$$
\operatorname{SINR}=\frac{P_{D}}{P_{I}+P_{N}}
$$

In the following, we investigate how the joint beamforming weights should be designed to mitigate the impact of Doppler spread due to high speed.
We define a vector parameter for the SIR optimisation problem as

$$
\boldsymbol{\omega}=\left[\begin{array}{l}
\mathbf{w}_{1} \\
\mathbf{w}_{0}
\end{array}\right]
$$

Let $\boldsymbol{\Theta}_{n}=\left[\begin{array}{ll}\mathbf{0}_{1 \times K} & \mathbf{H}_{n}\end{array}\right], \quad \boldsymbol{\Pi}_{n}=\left[\begin{array}{ll}\mathbf{H}_{n} & \mathbf{H}_{n}^{\prime}\end{array}\right]$, and select $t_{0}=1$, then the SINR can be reformulated as

$$
\begin{aligned}
\text { SINR } & =\frac{P_{D}}{P_{I}+P_{N}} \\
& =\frac{E\left\{\operatorname{tr}\left(\boldsymbol{\Theta}_{n} \boldsymbol{\omega} \boldsymbol{\omega}^{H} \boldsymbol{\Theta}_{n}^{H}\right)\right\}}{E\left\{\operatorname{tr}\left(\boldsymbol{\Pi}_{n} \boldsymbol{\omega} \boldsymbol{\omega} \boldsymbol{\omega}^{H} \Pi_{n}^{H}\right)\right\}+\sigma_{n}^{2}} \\
& =\frac{E\left\{\operatorname{tr}\left(\boldsymbol{\omega}^{H} \mathbf{R}_{\Theta} \boldsymbol{\omega}\right)\right\}}{E\left\{\operatorname{tr}\left(\boldsymbol{\omega}^{H} \mathbf{R}_{\Pi} \boldsymbol{\omega}\right)\right\}}
\end{aligned}
$$

where $\mathbf{R}_{\boldsymbol{\Theta}}=\boldsymbol{\Theta}_{n}^{H} \boldsymbol{\Theta}_{n}$ and $\mathbf{R}_{\boldsymbol{\Pi}}=\boldsymbol{\Pi}_{n}^{H} \boldsymbol{\Pi}_{n}$. Since $\mathbf{R}_{\boldsymbol{\Theta}}$ and $\mathbf{R}_{\boldsymbol{\Pi}}$ are both covariance matrices, they are Hermitian positive definitive.

Hence, optimising the SIR is a generalized Rayleigh quotient issue (p540 [19]), and the solution is the eigen vector corresponding to the maximum generalized eigen value of the matrix cluster $\left(\mathbf{R}_{\Theta}, \mathbf{R}_{\Pi}\right)$, e.g.

$$
\begin{aligned}
\boldsymbol{\omega} & =\arg \max _{\boldsymbol{\omega}}\left(\frac{E\left\{\operatorname{tr}\left(\boldsymbol{\omega}^{H} \mathbf{R}_{\boldsymbol{\Theta}} \boldsymbol{\omega}\right)\right\}}{E\left\{\operatorname{tr}\left(\boldsymbol{\omega}^{H} \mathbf{R}_{\Pi} \boldsymbol{\omega}\right)\right\}}\right) \\
& =\operatorname{Ve}\left\{\max \left\{\lambda\left(\mathbf{R}_{\boldsymbol{\Theta}}, \mathbf{R}_{\boldsymbol{\Pi}}\right)\right\}\right\} .
\end{aligned}
$$

where $\mathbf{R}_{\Theta}$ and $\mathbf{R}_{\Pi}$ can be obtained from the known channel correlation information, $\lambda(\mathbf{A}, \mathbf{B})$ represents the generalized eigen values of matrix cluster $(\mathbf{A}, \mathbf{B})$, and $\mathrm{V} e\{\bullet\}$ means taking the corresponding eigen vector.

\section{Numerical results}

In this section, we provide numerical results to demonstrate that our proposed cloud-based HSTC architecture in conjunction with the ICI cancellation algorithm based on transmit beamforming are capable of achieving 100 Mbps of data rate at a high speed of $450 \mathrm{kmph}$. We consider a C-HSTC system where each RRH is equipped with multiple directional antennas. By properly placing the antennas, a RRH covers both of its sides with radius $R$. Three adjacent RRHs are selected each time for serving 2 sufficiently separated TAUs, e.g. one equipped at the train front and the other at train end as illustrated in Figure 2. The 2 TAUs are configured with $20 \mathrm{MHz}$ of bandwidth centered around $2 \mathrm{GHz}$.

We model the mobility with a correlated Rayleigh channel generated based on an inverse Discrete Fourier Transform method [21]. The channel state information (CSI) is obtained at receiver via estimation based on reference signals. We assume a TDD system, thus the base station exploits channel reciprocity to obtain downlink channel state information. For OFDM channels from the RRHs two each TAU, we assume number of 
Table 1 Effective data rate of different MCSs and SNR requirements [22]

\begin{tabular}{cccc}
\hline MCS scheme & Input SINR for BER $=\mathbf{1 e - 5}(\mathbf{d B})$ & Effective bit rate (bits/symbol) & Data rate $(\mathbf{M b p s / T A U )}$ \\
\hline QPSK, $R=7 / 8$ & 4.7 & 1.75 & 32.615 \\
16QAM, $R=1 / 2$ & 6.8 & 2 & 37.274 \\
16QAM, $R=3 / 4$ & 7.0 & 3 & 55.911 \\
64QAM, $R=3 / 4$ & 10.6 & 4.5 & 83.867 \\
64QAM, $R=5 / 6$ & 11.9 & 5 & 93.185 \\
64QAM, $R=7 / 8$ & 12.8 & 5.25 & 97.844 \\
\hline
\end{tabular}

subcarriers $N=2048$. We also set the transmission interval (TTI) to 1 millisecond and each TTI consists of $M=14$ OFDM symbols. Therefore the OFDM symbol duration is 71.4 microseconds, which complies with the 3GPP LTE design [22]. The symbol rate of this system can be given by,

$$
R_{s}=(1-\gamma) N M / T_{T T I}
$$

where $\gamma$ is the signalling overhead, up to $35 \%$ for LTE systems [22]. Then, the calculated symbol rate is $R_{s}=18.637 \times 10^{6} \mathrm{symbols} / \mathrm{s}$. There are 4 pilot subcarriers for channel estimation in each resource block of 3 symbols $\times 4$ subcarriers. A minimum mean square error (MMSE) based receiver is employed at the receiver.

We adopt rate-compatible turbo codes for error controlling. The system bit rate is determined by the modulation and coding scheme (MCS), which is capable of providing satisfactory error performance with regard to a given input SINR. Table 1 shows the required input SINRs to the demodulator in order to achieve a bit error rate (BER) of $10^{-5}$, which is generally regarded as an 'error free' scenario for different MCSs.
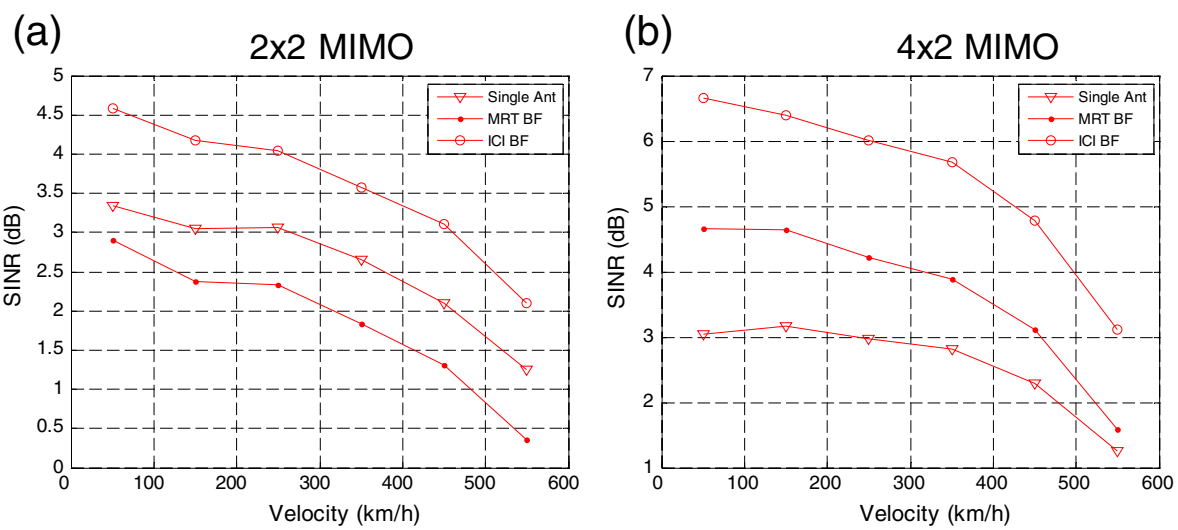

(c)

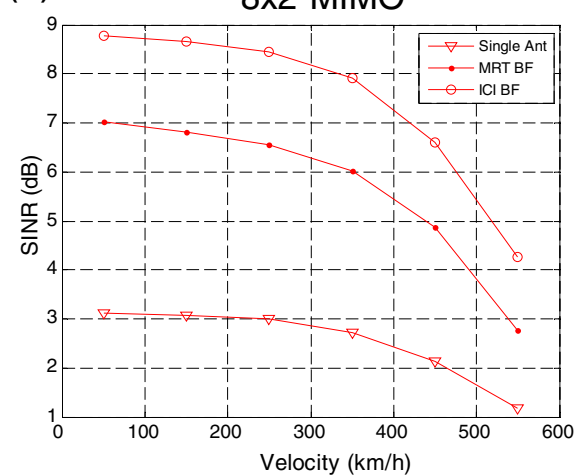

(d)
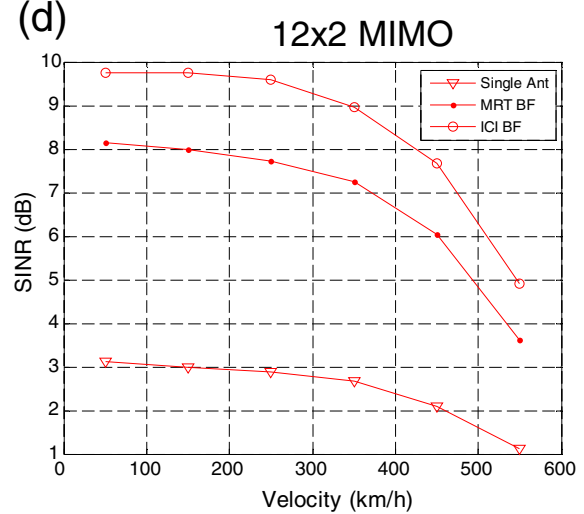

Figure 5 SINR performance for single RRH at SNR $=\mathbf{3 0} \mathbf{d B}$. (a) $\mathrm{SNR}=40 \mathrm{~dB}$ (b) $\mathrm{SNR}=30 \mathrm{~dB}(\mathbf{c}) \mathrm{SNR}=20 \mathrm{~dB}(\mathbf{d}) \mathrm{SNR}=10 \mathrm{~dB}$. 
From Table 1, in order to guarantee the peak data rate of $100 \mathrm{Mbps}$ for a user on a train equipped with 2 TAUs, the system should use 16QAM and $\mathrm{R}=3 / 4$ for modulation and coding, and guarantee a demodulator input SINR of $7.0 \mathrm{~dB}$. We will demonstrate how the system can achieve this SINR at the velocity of $450 \mathrm{kmph}$. For comparison, three transmission schemes are considered:

- Single Ant: Single antenna transmission and receiving system. In case of multiple RRHs, each $\mathrm{RRH}$ is equipped with one antenna.

- MRT BF: C-HSTC system with conventional Maximum Ratio Transmission (MRT) beamforming algorithm.

- ICI BF: C-HSTC system with the newly proposed ICI compensation via transmit beamforming algorithm.

We firstly experiment with different antenna configurations at both the RRH and the TAU sides for different channel signal-to-noise (SNR) ratios. For the RRH side we consider 2, 4, 8, and 12 antennas, while for the TAU side we consider 1 and 2 antennas. For fairness of comparison, the total transmission power of each $\mathrm{RRH}$ is fixed to $46 \mathrm{dBm}$, and the noise floor of each TAU is fixed to $-90 \mathrm{dBm}$.

Figure 5 shows the receiver output SINRs (equivalent to the demodulator input SINRs) versus the velocity under the different numbers of transmit antennas for three transmission schemes and the fixed channel SNR of $30 \mathrm{~dB}$.

The important observation is that the newly proposed ICI BF algorithm can provide a notable receiver output SINR gain, up to $2 \mathrm{~dB}$, over the conventional MRT BF approach. The reason is that MRT tries to maximize the signal-to-noise ratio. For the channel SNR of $30 \mathrm{~dB}$, the dominating factor for system performance becomes interferences due to Doppler shifts, e.g. inter-channelinterferences (ICI). At the velocity of $50 \mathrm{kmph}$, the channel can be accurately estimated, thus the ICI BF shows the best performance gain, while at the velocity of $450 \mathrm{~km} / \mathrm{h}$, the channel estimation becomes inaccurate, thus the performance of all configurations drops. But it is notable that the ICI BF algorithm still shows significant gain over the MRT BF.

It is also worth noting that conventional MRT BF cannot guarantee the required $7.0 \mathrm{~dB}$ SINR even when configured with 12 transmit antennas. However, the newly proposed ICI BF can guarantee this SINR at least for the
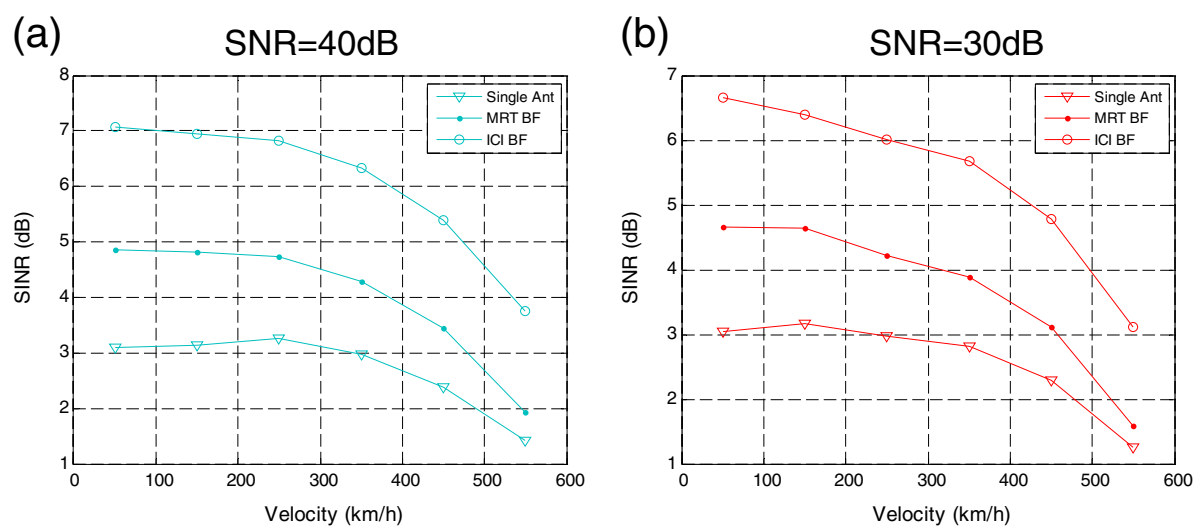

(c)

$\mathrm{SNR}=20 \mathrm{~dB}$

(d)
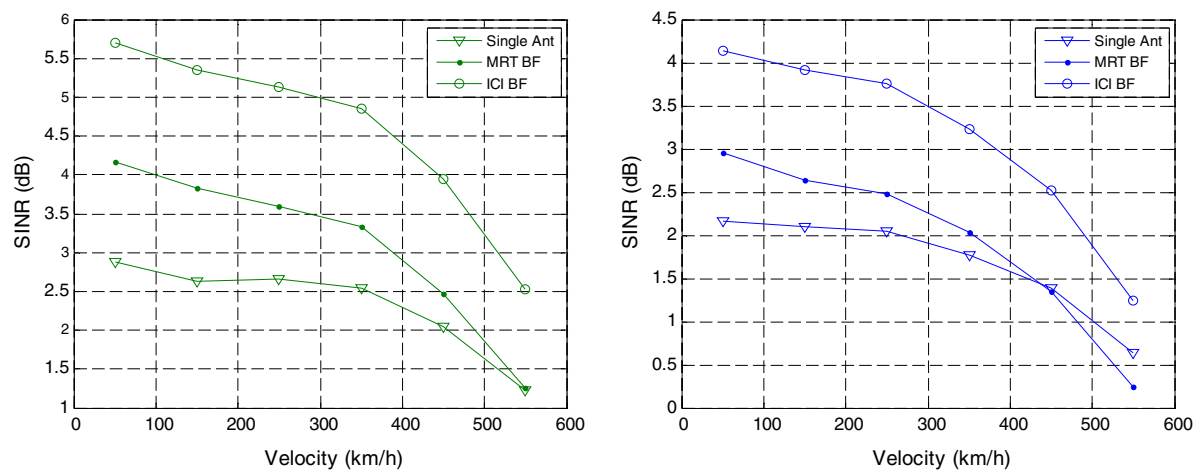

Figure 6 SINR performance for single RRH at $4 \times 2$ MIMO with different SNRs. 


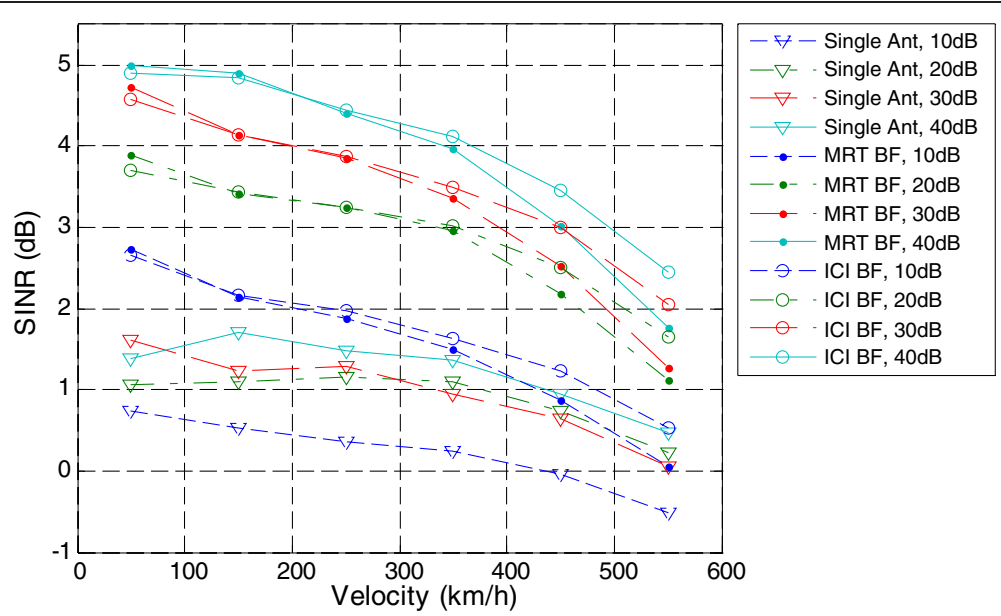

Figure 7 SINR performance for single RRH at $4 \times 1$ MIMO with different SNRs. (a) Radius $=800 \mathrm{~m}$ (b) Radius $=1600 \mathrm{~m}$ (c) Radius $=4000 \mathrm{~m}$ (d) Radius $=8000 \mathrm{~m}$.

$12 \times 2$ MIMO case. Therefore, for typical antenna configurations, the system with the proposed ICI BF achieves the best SINR performance in all of the three algorithms from low to high mobility. These results demonstrate that the proposed ICI BF algorithm could significantly improve performance of HSTC systems.

Figure 6 shows the effects of the change of channel SNR, corresponding to the change of transmission (a)

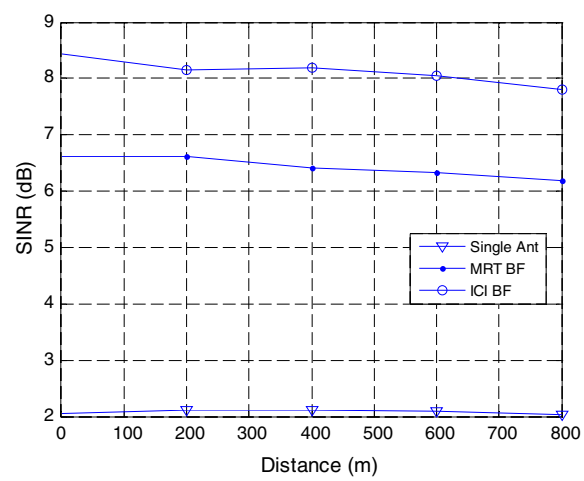

(c)

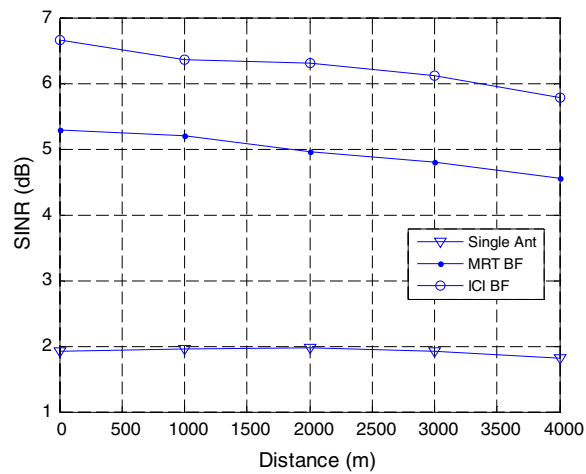

(b)

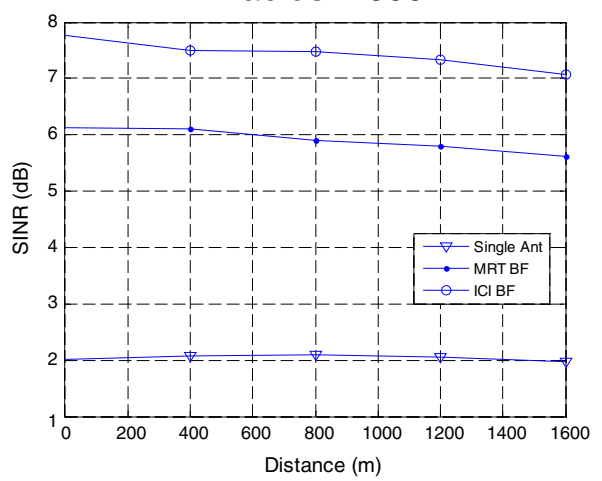

(d)

Radius $=8000 \mathrm{~m}$

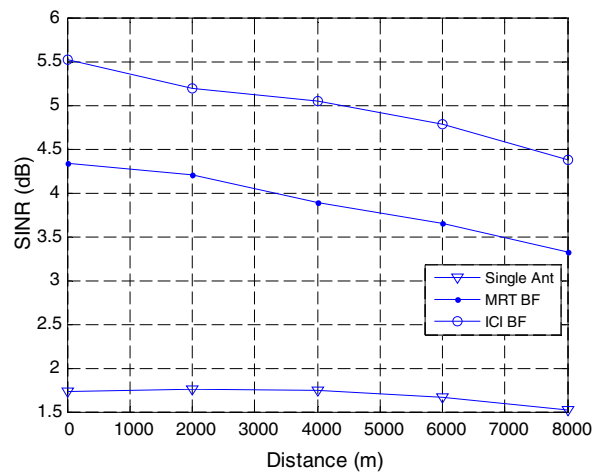

Figure 8 SINR performance for per RRH Pt $=46 \mathrm{dBm}$ and $\mathrm{Pn}=-90 \mathrm{dBm}, \mathrm{LOS}, 3$ adjacent RRHs selected for joint transmission, velocity $=450 \mathrm{kmph}$. 
power or transmission distance. We can see that with the increase of channel SNR, the ICI BF based system maintains steady SINR performance improvement compared to MRT BF and single antenna systems. In particular, with the decrease of the channel SNR, the ICI BF gain over MRT BF maintains, which demonstrates the advantage of our proposed algorithm.

Meanwhile, Figure 7 illustrates the receiver output SINR (demodulator input SINR) versus the velocity with different transmission algorithms and channel SNRs for the 4 transmit antennas and single receive antenna cases. We can see that the ICI BF still shows gain at high mobility over MRT BF, but the gain diminishes for medium to low mobility. This indicates that, in order to meet the target, each TAU should be equipped with at least 2 antennas.

Based on above experiments and observations, we simulate a C-HSTC system with a number of RRHs along the track and 2 TAUs on each train. Each RRH is equipped with 4 antennas, and each TAU is equipped with 2 antennas. The central scheduler selects 3 adjacent RRHs for joint beamforming each time. We employ the line of sight (LOS) path loss model [22] to simulate the large-scale signal attenuation due to propagation. The velocity of the train is fixed to 450 kilometers per hour.

Figure 8 illustrates the achievable SINRs at different locations relative to an RRH in the C-HSTC system with different transmission schemes.

When the radius increases from 800 meters to 8000 meters, the minimum receiver output SINRs decreases from $7.8 \mathrm{~dB}$ to $4.4 \mathrm{~dB}$ for ICI $\mathrm{BF}$, and from $6.2 \mathrm{~dB}$ to $3.3 \mathrm{~dB}$ for MRT BF. It is important to observe that, the conventional MRT BF cannot achieve the performance target of $7 \mathrm{~dB}$ output SINR even with the minimum 800 meter coverage radius. But by employing the newly proposed ICI BF algorithm, the $7.0 \mathrm{~dB}$ of demodulator output SINR can be guaranteed for the entire coverage with an RRH coverage radius as large as 1600 meters. For practical deployment, this significantly increased RRH coverage radius means more than $50 \%$ of RRH investment saving.

\section{Conclusions}

This paper proposes a C-HSTC framework for providing broadband services to highly mobile users. Within this framework, we propose a virtualized single cell design which mitigates the impact of conventional handover failure and guarantees continuous services. The virtualized single cell design also enables an efficient joint transmit beamforming scheme capable of delivering significant link level performance gain and providing sufficient data rate even at high mobility. We have demonstrated how to achieve 100 Mbps transmission at the speed of $450 \mathrm{kmph}$ for such a C-HSTC system framework.

The proposed framework may not only overcome the challenge of high call drop rate due to frequent handover failures at high mobility but also achieve quality broadband communications under strong ICI environment due to severe Doppler frequency shift in the scenario of high speed train communications. Generally, as the train speed increases and the location changes, the framework we propose can efficiently select the RRHs for service accessing and smoothly transport the user traffic to receivers with little change in user-perceived quality. We have demonstrated that the proposed framework and algorithm could be a strong candidate for high-speed train communications.

\section{Abbreviations}

3GPP: Third generation partnership project; BBU: Base band unit; BER: Bit error rate; BF: Beam forming; BS: Base station; CBTC: Communication-based train control; CPRI: Common public radio interface; $\mathrm{CRH}$ : China railway highspeed; CSI: Channel state information; GN: Ground network; HSTC: Highspeed train communication; ICl: Inter-channel interference and inter-career interference; LOS: Line of sight; LTE: Long term evolution; MAP: Multiplestandard accessing point; MIMO: Multiple input multiple output;

MRT: Maximum ratio transmission; OFDM: Orthogonal frequency division multiplexing; RAU: Radio access unit; RoF: Radio over fiber; RRH: Remote radio head; SINR: Signal to interference plus noise ratio; TAU: Train access unit; TTI: Transmission time interval.

\section{Competing interests}

The authors declare that they have no competing of interests.

\section{Acknowledgements}

The authors would like to acknowledge the contributions of our colleague Dr. Yang Tao for his advice on the architecture of the C-HSTC system as we prepared this manuscript for publication. This work was supported by the National Basic Research Program of China (973 Program No. 2012CB316100) and the NSFC under grant No.60872013/61032002.

\section{Author details}

${ }^{1}$ Bell Laboratories China, Room D400, Building 3, No. 388, Ningqiao Road, Pudong New District, Shanghai 201206, People's Republic of China. ${ }^{2}$ The Provincial Key Lab of Information Coding and Transmission, Southwest Jiaotong University, Chengdu, Sichuan 610031, People's Republic of China.

Received: 16 February 2012 Accepted: 8 August 2012

Published: 11 September 2012

\section{References}

1. http://en.wikipedia.org/wiki/GSM-R

2. H Guo, H Wu, Y Zhang, GSM-R Network Planning for High Speed Railway, in Proc. IET 3rd International Conference on Wireless, Mobile and Multimedia Networks (ICWMNN 2010) (Beijing, China, 26-29 Sep. 2010), pp. p10-13

3. B Lannoo, D Colle, M Pickavet, P Demeester, Radio-over-fibre-based solution to provide broadband internet access to train passengers. IEEE Commun Mag 45(2), 56-62 (2007)

4. FD Greve, B Lannoo, L Peters, TV Leeuwen, FV Quickenborne, D Colle, FD Turck, I Moerman, M Pickavet, B Dhoedt, P Demeester, FAMOUS: a network architecture for delivering multimedia services to fast moving users. Wirel. Personal Commun. J 33, 281-304 (2005)

5. C Ni, High-speed Railway Coverage Solution under SDR Solution. Design Technologies of Post and Telecommunications Systems, 18-21 (2009)

6. JY Zhang, ZH Tan, XX Yu, Coverage Efficiency of Radio-over-Fiber network for High-speed Railways, in Proc. Wireless Communications Networking and Mobile Computing (WiCOM 2010) (Chengdu, China,

Sep. 2010) 
7. JY Zhang, ZH Tan, ZD Zhong, Y Kong, A Multi-mode and Multi-systembased Access Architecture for High-speed Railways, in Proc. IEEE Vehicle Technologies Conference Fall (VTC 2010-Fall) (Ottawa, Canada, Sep. 2010)

8. CW Chow, YL Liu, SK Wen, SY Chen, CR Sheu, MC Tseng, JL Lin, DZ Hsu, S Chi, Theory and Technology for Standard WiMAX Over Fiber in High Speed Train Systems. Journal of Lightwave Technology 28(16), 2327-2336 (2010)

9. CPRI Specification, (2010)

10. O Andre, "LTE and Its Application in Railways", in Proc (Workshop on Networks and the New Economy, Cambridge, 2010)

11. M Wang, Y Yang, M Kazmi, A Larmo, J Pettersson, W Muller, Y Timner, Handover within 3GPP LTE: Design Principles and Performance, in Proc. IEEE Vehicular Technology Conference Fall (VTC 2009-Fall), Sept., 2009

12. 3GPP TS36.836, v1.0, (2012)

13. A Gorokhov, JP Linnartz, Robust OFDM receivers for dispersive time-varying channels: equalization and channel acquisition. IEEE Transaction on Communication 52(4), 572-583 (2004)

14. S Tomasin, A Gorokhov, H Yang, JP Linnartz, Iterative Interference Cancellation and Channel Estimation for Mobile OFDM. IEEE Trans Wirel Commun 4, 1 (2005)

15. A Seyedi, GJ Saulnier, General ICI self-cancellation scheme for OFDM systems. IEEE Transactions on Vehicle Technologies 54(1), 198-210 (2005)

16. S Serbetli, A Simple Antenna Combining Framework for Doppler Compensation in Mobile OFDM Systems, in Proc. IEEE International Conference on Acoustics, Speech and Signal Processing, Mar, 2008

17. YC Yu, M Okada, H Yamamoto, Study for various array antenna assisted Doppler spread compensator with MRC diversity of ISDB-T receiver, in Proc. IEEE Vehicular Technology Conference (VTC 2006), 2006

18. RD Pascoe, TN Eichorn, What is communication-based train control? IEEE Veh Technol Mag 4(4), 16-21 (2009)

19. X Zhang, Matrix Analysis and Applications (Springer, 2004)

20. G Dimic, ND Sidiropoulos, On downlink beamforming with greedy user selection: Performance analysis and a simple new algorithm. IEEE Trans. on Signal Processing 52(10), 3857-3868 (2005)

21. M Biguesh, AB Gershman, Downlink channel estimation in cellular systems with antenna arrays at base stations using channel probing with feedback. EURASIP J. Appl. Signal Process. (Special Issue on Advances in Smart Antennas) 9, 1330-1339 (2004)

22. 3GPP RAN1 TS36.211, Release 10, (2011)

doi:10.1186/1687-1499-2012-285

Cite this article as: Luo et al:: Reliable broadband wireless

communication for high speed trains using baseband cloud. EURASIP

Journal on Wireless Communications and Networking 2012 2012:285.

\section{Submit your manuscript to a SpringerOpen ${ }^{\circ}$ journal and benefit from:}

- Convenient online submission

- Rigorous peer review

- Immediate publication on acceptance

- Open access: articles freely available online

- High visibility within the field

- Retaining the copyright to your article

Submit your next manuscript at $\gg$ springeropen.com 